Proc. Indian Acad. Sci. (Math. Sci.), Vol. 105, No. 1, February 1995, pp. 23-29.

(C) Printed in India.

\title{
Flat connections, geometric invariants and energy of harmonic functions on compact Riemann surfaces
}

\author{
K GURUPRASAD \\ Department of Mathematics, Indian Institute of Science, Bangalore 560012, India \\ MS received 1 September 1993; revised 23 March 1994
}

\begin{abstract}
A geometric invariant is associated to the space of flat connections on a $G$-bundle over a compact Riemann surface and is related to the energy of harmonic functions.
\end{abstract}

Keywords. Principal G-bundle; flat connections; Chern-Simons forms; energy of maps; harmonic maps.

\section{Introduction}

This work grew out of an attempt to generalize the construction of Chern-Simons invariants. In this paper, we associate a geometric invariant to the space of flat connection on a $S U(2)$-bundle on a compact Riemann surface and relate it to the energy of harmonic functions on the surface.

Our set up is as follows. Let $G=S U(2)$ and $M$ be a compact Riemann surface and $E \rightarrow M$ be the trivial $G$-bundle. (Any $S U$ (2)-bundle over $M$ is topologically trivial). Let $\mathscr{C}$ be the space of all connections and $\mathscr{F}$ the subspace of all flat connections on this $G$-bundle. We endow on $\mathscr{C}$ the Frechet topology and the subspace topology on $\mathscr{F}$.

Given a loop $\sigma: S^{1} \rightarrow \mathscr{F}$, we can extend $\sigma$ to the closed unit disc $\tilde{\sigma}: D^{2} \rightarrow \mathscr{C}$ since $\mathscr{C}$ is contractible. On the trivial $G$-bundle $E \times D^{2} \rightarrow M \times D^{2}$ we define a tautological connection form $9^{\sigma}$ as follows

$$
\left.\vartheta^{\sigma}\right|_{(e, t)}=\tilde{\sigma}(t) \forall(e, t) \in E \times D^{2} .
$$

Clearly restriction of $\vartheta^{\sigma}$ to the bundle $E \times\{t\} \rightarrow M \times\{t\}$ is $\tilde{\sigma}(t) \forall t \in D^{2}$. Let $K\left(\vartheta^{\sigma}\right)$ be the curvature form of $\vartheta^{\sigma}$. Evaluation of the second Chern polynomial on this curvature form $K\left(9^{\sigma}\right)$ gives a closed 4-form on $M \times D^{2}$, which when integrated along $D^{2}$ yields a 2-form on $M$. This 2 -form is closed since $\operatorname{dim} M=2$ and thus defines an element in $H^{2}(M, R) \approx R$. It is seen that this class is independent of the extension of $\sigma$. We thus have a map

$$
\chi: \Omega(\mathscr{F}) \rightarrow H^{2}(M, R) \approx R
$$

where $\Omega(\mathscr{F})$ is the loop-space of $\mathscr{F}$.

We assume that the genus of $M \geqslant 2$. The energy $E(f)$ of any smooth function $f: M \rightarrow G$ is defined using the Poincare metric on $M$ and the bi-invariant metric on $\boldsymbol{G}=S U(2)$ given by the Killing form. 
Any smooth function $f: M \rightarrow G$ defines a flat connection $\omega_{f}=f^{*}(\mu)$ on the trivial bundle $M \times G \rightarrow M$, where $\mu$ is the Maurer-Cartan form on $G$. By a result of Hitchin ([H]), the loop in $\mathscr{C}$ is given by

$$
\sigma_{f}(t)=\frac{1}{2}\left(\omega_{f}+(\cos t) \omega_{f}+(\sin t)\left(* \omega_{f}\right)\right) \text { for } t \in[0,2 \pi]
$$

where $*: \Lambda^{1}(M, \mathscr{G}) \rightarrow \Lambda^{1}(M, \mathscr{G})$ is the Hodge star operator, is actually a loop in $\mathscr{F}$ if and only if $f$ is harmonic. ( $G$ is the Lie-Algebra of $G$ ).

The main result of this paper is

Theorem If $f: M \rightarrow G$ is a harmonic map, then

$$
\chi\left(\sigma_{f}\right)=-\frac{1}{4 \pi} E(f)
$$

\section{Construction of the basic geometric invariant}

In this paper we suppose $M$ is a compact Riemann surface of genus $g \geqslant 2, G=S U(2)$ with Lie algebra $\mathscr{G}=s u(2)$ and $\pi: E \rightarrow M$ is the trivial $G$-bundle on $M$. $\mathscr{C}$ is the space of connections and $\mathscr{F}$ is the subspace of all flat connections on $E \rightarrow M . D^{2}$ is the closed unit disc in $R^{2}$ and $\partial D^{2}=S^{1}$ is the unit circle. $\Omega(\mathscr{F})=\operatorname{Map}\left(S^{1}, \mathscr{F}\right)$ is the loop-space of $\mathscr{F}$. Given a loop $\sigma: S^{1} \rightarrow \mathscr{F}$ we extend $\sigma$ to $\tilde{\sigma}: D^{2} \rightarrow \mathscr{C}$ ( $\mathscr{C}$ is contractible). On the trivial bundle $E \times D^{2} \rightarrow M \times D^{2}$, let $9^{\sigma}$ be the tautological connection defined in the introduction. Let $K\left(\vartheta^{\sigma}\right)$ be the curvature 2-form of the connection $\vartheta^{\sigma}$. Let $C_{2}$ be the second Chern polynomial on $\mathscr{G}$. For the Lie algebra $\mathscr{G}=s u(2), C_{2}$ is essentially the determinant. More particularly $C_{2}(A)=-\left(1 / 4 \pi^{2}\right) \operatorname{det}(A)$ for $A \in \operatorname{su}(2)$ (cf. [KN], Chap. XII). Now an easy computation shows that

$$
C_{2}(A)=\frac{1}{8 \pi^{2}} \operatorname{trace}\left(A^{2}\right) \text { for } A \in \mathscr{G}
$$

Evaluation of $C_{2}$ on $K\left(\vartheta^{\sigma}\right)$ gives a closed 4-form $\overline{C_{2}\left(K\left(\vartheta^{\sigma}\right)\right)}$ on $E \times D^{2}$ which projects to a closed 4-form $C_{2}\left(K\left(\vartheta^{\sigma}\right)\right)$ on $M \times D^{2}$. Integrating $C_{2}\left(K\left(\vartheta^{\sigma}\right)\right)$ along $D^{2}$ yields a closed 2-form on $M(\operatorname{dim} M=2)$ and thus defines a cohomology class in $H^{2}(M, R)$ i.e.

$$
\left\{\int_{D^{2}} C_{2}\left(K\left(\vartheta^{\sigma}\right)\right)\right\} \in H^{2}(M, R) \approx \mathrm{R} .
$$

We outline the proof of the following lemma (cf. [G], §1 and [GS], §2,3).

Lemma 1.1. $\int_{D^{2}} C_{2}\left(K\left(\vartheta^{\sigma}\right)\right)$ is independent of the extension of $\sigma: S^{1} \rightarrow \mathscr{C}$ to $\tilde{\sigma}: D^{2}: \rightarrow \mathscr{C}$.

Proof. Let $\tilde{\sigma}_{1}, \tilde{\sigma}_{2}$ be two extensions of $\sigma$ with corresponding connection forms $\vartheta_{1}^{\sigma}, \vartheta_{2}^{\sigma}$ and curvature forms $K\left(\vartheta_{1}^{\sigma}\right), K\left(\vartheta_{2}^{\sigma}\right)$ on the bundle $E \times D^{2} \rightarrow M \times D^{2}$. On $E \times D^{2}$ we have

$$
\begin{aligned}
& \mathrm{d} T C_{2}\left(\vartheta_{1}^{\sigma}\right)=\overline{C_{2}\left(K\left(\vartheta_{1}^{\sigma}\right)\right)} \\
& \mathrm{d} T C_{2}\left(\vartheta_{1}^{\sigma}\right)=\overline{C_{2}\left(K\left(\vartheta_{2}^{\sigma}\right)\right)}
\end{aligned}
$$


where $T C_{2}\left(\vartheta_{1}^{\sigma}\right), T C_{2}\left(\vartheta_{2}^{\sigma}\right)$ are the Chern-Simons secondary forms with respect to $\vartheta_{1}^{\sigma}, \vartheta_{2}^{\sigma}$ respectively (cf. [CS, 3]). We can easily check that $\overline{C_{2}\left(K\left(\vartheta_{1}^{\sigma}\right)\right)}-\overline{C_{2}\left(K\left(\vartheta_{2}^{\sigma}\right)\right)}$ is an exact form on $E$ (cf. $[\mathrm{G}, 1]$ ). Since $\pi^{*}: H^{2}(M, R) \rightarrow H^{2}(E, R)$ is an isomorphism it follows that $\left\{C_{2}\left(K\left(\vartheta_{1}^{\sigma}\right)\right)\right\}=\left\{C_{2}\left(K\left(\vartheta_{1}^{\sigma}\right)\right)\right\} \in H^{2}(M, R)$ and this proves the lemma.

We thus have a map

$$
\begin{aligned}
& \Omega(\mathscr{F}) \stackrel{\chi}{\rightarrow} H^{2}(M, R) \approx R \\
& \sigma \mapsto \chi(\sigma)=\left\{\int_{D^{2}} C_{2}\left(K\left(\vartheta^{\sigma}\right)\right)\right\}
\end{aligned}
$$

where $\Omega(\mathscr{F})$ is the loop-space of $\mathscr{F}$. It is easy to check that $\chi\left(\sigma \circ \sigma^{\prime}\right)=\chi(\sigma)+\chi\left(\sigma^{\prime}\right)$ where $\sigma \circ \sigma^{\prime}$ is the composite of two loops in $\mathscr{F}$. We call this map $\chi$ the geometric invariant.

\section{Energy of functions and a class of special loops}

We recall the definition of energy of a function. Let $X$ and $Y$ be Riemannian manifolds. Given a smooth map $f: X \rightarrow Y$, the energy density of $f$ is a function $e(f): X \rightarrow R$ defined by

$$
e(f)(x)=\|\mathrm{d} f(x)\|^{2}
$$

where $\|\mathrm{d} f(x)\|$ denotes the Hilbert-Schmidt norm of the differential $\mathrm{d} f(x) \in T_{x}^{*}(x) \otimes$ $T_{f(x)}(Y)$. If $X$ is compact and oriented, the energy of $f$, denoted by $E(f)$ is given by

$$
E(f)=\left(\int_{M} e(f)(x) \mathrm{d} x\right)^{1 / 2}
$$

where $\mathrm{d} x$ is the volume form of $X$ with respect to its Riemannian metric. $f$ is harmonic if it is a critical point of the energy functional.

Using the Poincare metric on the compact Riemann surface of genus $\geqslant 2$ and the bi-invariant metric on $G=S U(2)$ given by the Killing form, we can define the energy $E(f)$ of a smooth function $f: M \rightarrow G$ by the above formula.

Any smooth function $f: M \rightarrow G$ defines a flat connection $\omega_{f}=f^{*}(\mu)$ on the trivial bundle $E \rightarrow M$ where

$$
\mu=\left(\begin{array}{cc}
i \mu_{1} & \mu_{2}+i \mu_{3} \\
-\mu_{2}+i \mu_{3} & -i \mu_{1}
\end{array}\right)
$$

is the Maurer-Cartan form on $G$. In the case of the trivial bundle $E \rightarrow M$, clearly the space of all connections $\mathscr{C}$ can be.identified with the space $\Lambda^{1}(M, \mathscr{G})$ of all $\mathscr{G}$-valued 1 -forms on $M$. For any smooth function $f: M \rightarrow G$, consider the loop in $\mathscr{C}$ given by $\sigma_{f}(t)=\frac{1}{2}\left(\omega_{f}+(\cos t) \omega_{f}+(\sin t)\left(* \omega_{f}\right)\right)$ for $t \in[0,2 \pi]$, where $*: \Lambda^{1}(M, \mathscr{G}) \rightarrow \Lambda^{1}(M, \mathscr{G})$ is the Hodge star operator. By a result of Hitchin $([\mathrm{H}])$, we know that $\sigma_{f}([0,2 \pi]) \subset \mathscr{F}$ iff $f$ is harmonic, i.e. $\sigma_{f}$ is a loop in $\mathscr{F}$ iff $f$ is harmonic. 


\section{Relation between the geometric invariant and the energy of harmonic maps}

We prove the following result

Theorem 3.1. If $f: M \rightarrow G$ is a harmonic map, then $\chi\left(\sigma_{f}\right)=-\frac{1}{4 \pi} E(f)$.

Proof. At the outset we show that the closed 2-form which represents $\chi\left(\sigma_{f}\right) \in H^{2}(M, R)$ is $\frac{1}{2 \pi}\left(* \omega_{1} \Lambda \omega_{1}+* \omega_{2} \Lambda \omega_{2}+* \omega_{3} \Lambda \omega_{3}\right)$ where

$$
\omega_{f}=f^{*} \mu=\left(\begin{array}{cc}
i \omega_{1} & \omega_{2}+i \omega_{3} \\
-\omega_{2}+i \omega_{3} & -i \omega_{1}
\end{array}\right)
$$

We extend the loop $\sigma_{f}$ in $\mathscr{F}$ to a map $\tilde{\sigma}_{f}: D^{2} \rightarrow \mathscr{C}$ in an obvious way. We drop the suffix $f$ and simply use $\sigma$ and $\tilde{\sigma}$ in the computations that follow.

Let $(s, t)$ be the polar coordinates on $D^{2}=\{(s, t), 0 \leqslant s \leqslant 1,0 \leqslant t \leqslant 2 \pi\}$.

Set $\tilde{\sigma}(s, t)=s \sigma(t)$. We now compute the curvature $K\left(\vartheta^{\sigma}\right)$ of the connection form $\vartheta^{\sigma}$ on the bundle $E \times D^{2} \rightarrow M \times D^{2}$.

$$
\begin{aligned}
K\left(\vartheta^{\sigma}\right) & =d \vartheta^{\sigma}+\frac{1}{2}\left[\vartheta^{\sigma}, \vartheta^{\sigma}\right] \\
& =d \vartheta^{\sigma}+\vartheta^{\sigma} \wedge \vartheta^{\sigma} \\
& =d_{E} \vartheta^{\sigma}+d_{D^{2}} \vartheta^{\sigma}+\vartheta^{\sigma} \wedge \vartheta^{\sigma}, \\
& =d_{D^{2}}^{9^{\sigma}}+K(\tilde{\sigma}(s, t)),
\end{aligned}
$$

where $K(\tilde{\sigma}(s, t)$ is the curvature of $\tilde{\sigma}(s, t))$ and $d_{E}$ and $d_{D^{2}}$ are respectively the exterior differentials on $E$ and $D^{2}$.

If we set

$$
\sigma(t)=\left(\begin{array}{cc}
i \alpha(t) & \beta(t)+i \gamma(t) \\
-\beta(t)+i \gamma(t) & -i \alpha(t)
\end{array}\right)
$$

as a form on $M$ for each $t \in S^{1}$, then after a straightforward calculation (see [G], Lemma 4.1), it follows that $\int_{D^{2}} C_{2}\left(K\left(\vartheta^{\sigma}\right)\right)$ is cohomologous to the form

Now

$$
\frac{1}{4 \pi^{2}} \int_{S^{1}}\left(\frac{\mathrm{d}}{\mathrm{d} t} \alpha(t) \wedge \alpha(t)+\frac{\mathrm{d}}{\mathrm{d} t} \beta(t) \wedge \beta(t)+\frac{\mathrm{d}}{\mathrm{d} t} \gamma(t) \wedge \gamma(t)\right) \mathrm{d} t
$$

so that

$$
\omega=f^{*} \mu=\left(\begin{array}{cc}
i \omega_{1} & \omega_{2}+i \omega_{3} \\
-\omega_{2}+i \omega_{3} & -i \omega_{1}
\end{array}\right)
$$

$$
\sigma(t)=\left(\begin{array}{cc}
i\left(\omega_{1}+\cos t \omega_{1}+\sin t * \omega_{1}\right) & \left(\omega_{2}+\cos t \omega_{2}+\sin t * \omega_{2}\right)+ \\
-\left(\omega_{2}+\cos t \omega_{2}+\sin t * \omega_{2}\right)+ & -i\left(\omega_{3}+\cos t \omega_{3}+\sin t * \omega_{3}\right) \\
i\left(\omega_{3}+\cos t \omega_{3}+\sin t * \omega_{3}\right) &
\end{array}\right)
$$


i.e.

Now

$$
\begin{aligned}
& \alpha(t)=\left(\omega_{1}+\cos t \omega_{1}+\sin t * \omega_{1}\right) \\
& \beta(t)=\left(\omega_{2}+\cos t \omega_{2}+\sin t * \omega_{2}\right) \\
& \gamma(t)=\left(\omega_{3}+\cos t \omega_{3}+\sin t * \omega_{3}\right)
\end{aligned}
$$

$$
\begin{aligned}
\frac{\mathrm{d}}{\mathrm{d} t} \alpha(t) \Lambda \alpha(t) & =\left((-\sin t) \omega_{1}+\cos t * \omega_{1}\right) \Lambda\left(\omega_{1}+\cos t * \omega_{1}+\sin t * \omega_{1}\right) \\
& =-\sin ^{2} t \omega_{1} \Lambda * \omega_{1}+\cos ^{2} t * \omega_{1} \Lambda \omega_{1} \\
& =* \omega_{1} \Lambda \omega_{1} .
\end{aligned}
$$

Similarly

$$
\begin{aligned}
& \frac{\mathrm{d}}{\mathrm{d} t} \beta(t) \wedge \beta(t)=* \omega_{2} \wedge \omega_{2} \\
& \frac{\mathrm{d}}{\mathrm{d} t} \gamma(t) \wedge \gamma(t)=* \omega_{3} \wedge \omega_{3} .
\end{aligned}
$$

It follows that $\int_{D^{2}} C_{2}\left(K\left(\vartheta^{\sigma}\right)\right)$ is cohomologous to the form

$$
\begin{gathered}
\frac{1}{4 \pi^{2}} \int_{S^{1}}\left(* \omega_{1} \wedge \omega_{1}+* \omega_{2} \wedge \omega_{2}+* \omega_{3} \wedge \omega_{3}\right) d t \\
=\frac{1}{2 \pi}\left(* \omega_{1} \wedge \omega_{1}+* \omega_{2} \wedge \omega_{2}+* \omega_{3} \wedge \omega_{3}\right) .
\end{gathered}
$$

Thus the closed 2 -form on $M$ representing $\chi\left(\sigma_{f}\right) \in H^{2}(M, R)$ is $\frac{1}{2 \pi}\left(* \omega_{1} \wedge \omega_{1}+\right.$ $\left.* \omega_{2} \wedge \omega_{2}+* \omega_{3} \wedge \omega_{3}\right)$.

To prove that $\chi\left(\sigma_{f}\right)=-\frac{1}{4 \pi} E(f)$, we check using local coordinates that the forms

$$
\frac{1}{2 \pi}\left(\frac{\mathrm{d}}{\mathrm{d} t} \alpha(t) \wedge \alpha(t)+\frac{\mathrm{d}}{\mathrm{d} t} \beta(t) \wedge \beta(t)+\frac{\mathrm{d}}{\mathrm{d} t} \gamma(t) \wedge \gamma(t)\right)
$$

and $-\frac{1}{4 \pi} e(f)(m) \mathrm{d} m(\mathrm{~d} m$ is the volume form on $M)$ are equal at any arbitrary point.

Since any left translation in $G$ is an isometry, for any $m \in M,\|\mathrm{~d} f(m)\|=$ $\left\|\mathrm{d}\left(L_{f(m)^{-1}} \circ f\right)(m)\right\|$ where $L_{f(m)^{-1}}: G \rightarrow G$ is left translation by $f(m)^{-1}$. We can therefore assume that $f$ maps some point $m \in M$ to the identity element in $G$, i.e. $f(m)=1$.

Since we intend to use local coordinates to prove the equality of forms, we can go to the universal cover $D^{2}$ of $M$ with Poincare metric and assume $f: D^{2} \rightarrow G$ and $f(m)=1$ for some fixed $m \in D^{2}$. Since there exist an isometry of $D^{2}$ which maps the origin to $m$, we can assume $f(0)=1$ and check equality of forms at the origin.

At the origin we have

$$
\left\langle\frac{\partial}{\partial x}, \frac{\partial}{\partial x}\right\rangle=1=\left\langle\frac{\partial}{\partial y}, \frac{\partial}{\partial y}\right\rangle
$$


and

$$
\left\langle\frac{\partial}{\partial x}, \frac{\partial}{\partial y}\right\rangle=0
$$

where $\frac{\partial}{\partial x}, \frac{\partial}{\partial y}$ are the usual coordinate vector fields. Let $\mathrm{d} x$ and $\mathrm{d} y$ be the dual 1 -forms.

Clearly at the origin $* d x=d y$ and $* d y=-d x$. Since $d m=d x \wedge d y$ we have

$$
e(f)(m) \mathrm{d} m\left(\frac{\partial}{\partial x}, \frac{\partial}{\partial y}\right)=e(f)(m)
$$

We prove that

$$
\frac{1}{2 \pi}\left(* \omega_{1} \wedge \omega_{1}+* \omega_{2} \wedge \omega_{2}+* \omega_{3} \wedge \omega_{3}\right)\left(\frac{\partial}{\partial x}, \frac{\partial}{\partial y}\right)=-\frac{1}{4 \pi} e(f)(m) .
$$

If $\omega_{j}=a_{j} \mathrm{~d} x+b_{j} \mathrm{~d} y\left(1 \leqslant j \leqslant 3, a_{j}, b_{j}\right.$ are functions on $\left.D^{2}\right)$ then $* \omega_{j}=a_{j} \mathrm{~d} y-b_{j} \mathrm{~d} x$ for $1 \leqslant j \leqslant 3$ so that $* \omega_{j} \wedge \omega_{j}=-\left(a_{j}^{2}+b_{j}^{2}\right) \mathrm{d} x \wedge \mathrm{d} y$ for $1 \leqslant j \leqslant 3$

$\Rightarrow \frac{1}{2 \pi}\left(* \omega_{1} \wedge \omega_{1}+* \omega_{2} \wedge \omega_{2}+* \omega_{3} \wedge \omega_{3}\right)=-\frac{1}{2 \pi}\left(a_{1}^{2}+b_{1}^{2}+a_{2}^{2}+b_{2}^{2}+a_{3}^{2}+b_{3}^{2}\right) \mathrm{d} x \wedge \mathrm{d} y$

For $f: D^{2} \rightarrow S U(2)$ with $f(0)=1$

$$
\begin{aligned}
\|\mathrm{d} f(0)\|^{2} & =\left\|\mathrm{d} f(0)\left(\frac{\partial}{\partial x}\right)\right\|^{2}+\left\|\mathrm{d} f(0)\left(\frac{\partial}{\partial y}\right)\right\|^{2} \\
& =\left\|\frac{\partial f(0)}{\partial x}\right\|^{2}+\left\|\frac{\partial f(0)}{\partial y}\right\|^{2}
\end{aligned}
$$

By definition of Maurer-Cartan form

$$
\left.\begin{array}{ll}
\frac{\partial f(0)}{\partial x}=\mu\left(\frac{\partial f(0)}{\partial x}\right)= & \mu_{2}\left(\frac{\partial f(0)}{\partial x}\right)+i \mu_{3}\left(\frac{\partial f(0)}{\partial x}\right) \\
i \mu_{1}\left(\frac{\partial f(0)}{\partial x}\right) & -i \mu_{1}\left(\frac{\partial f(0)}{\partial x}\right)
\end{array}\right)
$$

The pairing $(A, B) \mapsto \operatorname{trace}(A B)$ for $A, B \in s u(2)$ gives the Killing form on $s u(2)$ so that

Similarly

$$
\begin{aligned}
\left\|\frac{\partial f(0)}{\partial x}\right\|^{2} & =\operatorname{trace}\left(\frac{\partial f(0)}{\partial x} \frac{\partial f(0)}{\partial x}\right) \\
& =2\left\{\left(\mu_{1}\left(\frac{\partial f(0)}{\partial x}\right)\right)^{2}+\left(\mu_{2}\left(\frac{\partial f(0)}{\partial x}\right)\right)^{2}+\left(\mu_{3}\left(\frac{\partial f(0)}{\partial x}\right)\right)^{2}\right\} .
\end{aligned}
$$

$$
\begin{aligned}
\left\|\frac{\partial f(0)}{\partial x}\right\|^{2} & =2\left\{\left(\mu_{1}\left(\frac{\partial f(0)}{\partial y}\right)\right)^{2}+\left(\mu_{2}\left(\frac{\partial f(0)}{\partial y}\right)\right)^{2}+\left(\mu_{3}\left(\frac{\partial f(0)}{\partial y}\right)\right)^{2}\right\} . \\
& \Rightarrow\|\mathrm{d} f(0)\|^{2}=2\left\{\sum_{j=1}^{3}\left(\mu_{j}\left(\frac{\partial f(0)}{\partial x}\right)\right)^{2}+\left(\mu_{j}\left(\frac{\partial f(0)}{\partial y}\right)\right)^{2}\right\} .
\end{aligned}
$$


Noting that $f^{*} \mu_{j}=\omega_{j}(1 \leqslant j \leqslant 3)$ we have

Now

$$
\omega_{j}\left(\frac{\partial}{\partial x}\right)(0)=\left(f^{*} \mu_{j}\right)\left(\frac{\partial}{\partial x}\right)(0)=\mu_{j}\left(\frac{\partial f}{\partial x}(0)\right) .
$$

Therefore

$$
\omega_{j}\left(\frac{\partial}{\partial x}\right)=\left(a_{j} \mathrm{~d} x+b_{j} \mathrm{~d} y\right)\left(\frac{\partial}{\partial x}\right)=a_{j}
$$

Similarly

$$
\omega_{j}\left(\frac{\partial f(0)}{\partial x}\right)=a_{j} \quad(1 \leqslant j \leqslant 3)
$$

$$
\omega_{j}\left(\frac{\partial f(0)}{\partial y}\right)=b_{j} \quad(1 \leqslant j \leqslant 3)
$$

Thus

$$
\|\mathrm{d} f(0)\|^{2}=2\left\{a_{1}^{2}+b_{1}^{\dot{2}}+a_{2}^{2}+b_{2}^{2}+a_{3}^{2}+b_{3}^{2}\right\}=e(f)(m) .
$$

Therefore we have

$$
\left(-\left(* \omega_{1} \wedge \omega_{1}+* \omega_{2} \wedge \omega_{2}+* \omega_{3} \wedge \omega_{3}\right)\right)=\frac{1}{2} e(f)(m) \mathrm{d} x \wedge \mathrm{d} y .
$$

In other words

$$
\left(\frac{1}{2 \pi}\left(* \omega_{1} \wedge \omega_{1}+* \omega_{2} \wedge \omega_{2}+* \omega_{3} \wedge \omega_{3}\right)\right)=-\frac{1}{4 \pi} e(f)(m) \mathrm{d} m
$$

Consequently $\chi\left(\sigma_{f}\right)=-\frac{1}{4 \pi} E(f)$ and the theorem follows.

\section{Acknowledgement}

It is a pleasure to thank A R Aithal, I Biswas and N Hitchin for helpful discussions.

\section{References}

[CS] Chern S S and Simons J, Characteristics forms and geometric invariants. Ann. Math. $9948-49$ (1974)

[G] Guruprasad K, Flat connections, geometric invariants and the simplectic nature of the fundamental group of surfaces. Pac. J. Math. 162 no. 1, (1994)

[GS] Guruprasad $K$ and Shrawan Kumar, $A$ new geometric invariant associated to the space of flat connections. Compos. Math. 73 199-222 (1990)

[H] Hitchin N J, Harmonic maps from 2-torus to the 3-sphere. J. Differ. Geom. 31, 627-710 (1990)

[KN] Kobayashi S and Nomizu K, Foundations of Differential Geometry, Vol. II Interscience Publications, (1969) 